\title{
Development and Characterization of a Novel Hybrid Tissue Engineering-Based Scaffold for Spinal Cord Injury Repair
}

\author{
Nuno A. Silva, B.Sc., ${ }^{1-3}$ Antonio J. Salgado, Ph.D., ${ }^{3}$ Rui A. Sousa, Ph.D., ${ }^{1,2}$ Joao T. Oliveira, Ph.D., ${ }^{1,2}$ \\ Adriano J. Pedro, B.Sc., ${ }^{1,2}$ Hugo Leite-Almeida, B.Sc., ${ }^{3}$ Rui Cerqueira, M.D., ${ }^{3}$ Armando Almeida, Ph.D., ${ }^{3}$ \\ Fabrizio Mastronardi, Ph.D., ${ }^{4}$ João F. Mano, Ph.D., ${ }^{1,2}$ Nuno M. Neves, Ph.D., ${ }^{1,2}$ \\ Nuno Sousa, Ph.D., ${ }^{3}$ and Rui L. Reis, Ph.D., ${ }^{1,2}$
}

Spinal cord injury (SCI) represents a significant health and social problem, and therefore it is vital to develop novel strategies that can specifically target it. In this context, the objective of the present work was to develop a new range of three-dimensional (3D) tubular structures aimed at inducing the regeneration within SCI sites. Up to six different 3D tubular structures were initially developed by rapid prototyping: 3D bioplotting-based on a biodegradable blend of starch. These structures were then further complemented by injecting Gellan Gum, a polysaccharide-based hydrogel, in the central area of structures. The mechanical properties of these structures were assessed using dynamic mechanical analysis, under both dry and wet conditions, and their morphologies/ porosities were analyzed using micro-computed tomography and scanning electron microscopy. Biological evaluation was carried out to determine their cytotoxicity, using both minimum essential medium (MEM) extraction and MTS tests, as well as by encapsulation of an oligodendrocyte-like cell (M03-13 cell line) within the hydrogel phase. The histomorphometric analysis showed a fully interconnected network of pores with porosity ranging from $70 \%$ to $85 \%$. Scaffolds presented compressive modulus ranging from 17.4 to $62.0 \mathrm{MPa}$ and 4.42 to

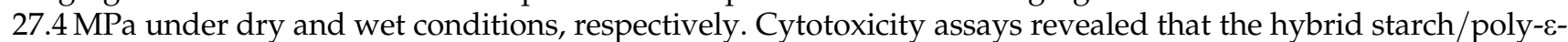
caprolactone/Gellan Gum scaffolds were noncytotoxic, as they did not cause major alterations on cell morphology, proliferation, and metabolic viability. Moreover, preliminary cell encapsulation assays showed that the hybrid scaffolds could support the in vitro culture of oligodendrocyte-like cells. Finally, preliminary in vivo studies conducted in a hemisection rat SCI model revealed that the above-referred structures were well integrated within the injury and did not trigger chronic inflammatory processes. The results herein presented indicate that these 3D systems might be of use in future SCI regeneration approaches.

\section{Introduction}

S Pinal CORD injury (SCI) results in a devastating con$\checkmark$ dition with enormous financial, ${ }^{1-4}$ social, and personal costs. ${ }^{5}$ It is estimated that the annual incidence of SCI in the United States alone is approximately 40 cases per 1 million. It is commonly characterized by a primary injury that leads to a cascade of cellular and biochemical reactions that cause further damage. The latter is known as "secondary injury," which is characterized by microvascular alterations, edema, ischemia, necrosis, free radicals formation, lipid peroxidation, excitatory neurotransmitter accumulation, inflamma- tory response, and other molecular changes contributing to further neural damage. ${ }^{2,6-9}$

Current approaches used in clinical practice are mainly based on the use of pharmacological agents, like methylprednisolone. ${ }^{10,11}$ However, its use is quite controversial, as recent studies failed to reveal conclusive beneficial outcomes. $^{12,13}$ Therefore, it is imperative to find novel therapeutic strategies that can specifically target SCI regeneration.

In recent years, different approaches have been proposed to develop valid strategies for SCI repair such as biomolecular, $^{14-17}$ cellular, ${ }^{18-23}$ and biomaterial-based therapies. ${ }^{24-28}$ However, due to the complexity of SCI repair it is unlikely

\footnotetext{
${ }^{1} 3 \mathrm{~B}$ 's Research Group-Biomaterials, Biodegradables, and Biomimetics, University of Minho, Headquarters of the European Institute of Excellence on Tissue Engineering and Regenerative Medicine, Guimarães, Portugal.

${ }^{2}$ Institute for Biotechnology and Bioengineering, PT Government Associated Lab, Guimarães, Portugal.

${ }^{3}$ Life and Health Sciences Research Institute (ICVS), School of Health Sciences, University of Minho, Braga, Portugal.

${ }^{4}$ Program in Molecular Structure and Function, The Hospital for Sick Children, Toronto, Canada.
} 
that a single strategy will be adequate to tackle the problem. It is probable that only by following an integrated strategy, such as that presented by tissue engineering, ${ }^{29}$ it will be possible to develop a successful approach. In this field, it is of general agreement that a scaffold, a three-dimensional (3D) biodegradable structure, plays a central role in the aid of the regenerative process. In recent years, natural-based biodegradable polymers have emerged as a possible biomaterial source for scaffold development within the SCI field. ${ }^{28,30-34}$ Among these, polysaccharides are particularly appealing owing to their structural similarities to components of host tissues. Moreover, they can also be enzymatically degraded in biological systems, allowing for better control of endogenous degradation rate along with other properties. ${ }^{35,36}$

Within the present study we aimed at developing a new bi-phasic tubular scaffold for SCI regeneration. It is comprised of two natural polysaccharide-based biomaterials: starch/poly-e-caprolactone (SPCL) blend and gellan gum. SPCL has been originally proposed by Reis and colleagues for a wide range of applications, including the culture of neurons, astrocytes, and oligodendrocytes. ${ }^{37-43}$ Gellan gum is an anionic exocellular polysaccharide secreted from the Sphingomonas paucimobilis bacterium. It is composed of repeated tetrasaccharide units of glucose, glucuronic acid and rhamnose residues in a 2:1:1 ratio. ${ }^{44}$ It was originally used as a thickener/gelling agent in food applications, and was recently proposed as a hydrogel for regenerative medicine purposes. ${ }^{44}$ The hybrid structures proposed in this report consist of an SPCL semi-rigid tubular porous structure filled by a gellan gum hydrogel concentric core. In this concept the SPCL tubular structure assures mechanical stability to the entire construct, namely, by establishing a connection to the adjacent vertebral bone. In this sense the SPCL phase is aimed at mimicking the bone functions, while the gellan gum hydrogel is used as a cell encapsulation system to support axonal regeneration in the injured spinal cord.

In this report we address the mechanical and preliminary biological characterization of SPCL/gellan gum hybrid tubular structures. Our results reveal that these novel structures exhibit adjustable mechanical performance and porosity with minimal cytotoxicity and support the encapsulation of oligodendrocyte-like cells. Moreover, it was also observed that these 3D hybrid structures caused a minimal inflammatory reaction when implanted subcutaneously and in a hemisection SCI model in Wistar rats.

\section{Materials and Methods}

\section{Materials}

The materials used in this work were a 30/70 (wt\%) biodegradable blend of SPCL and a polysaccharide gellan gum hydrogel 1\% (w/v) (Sigma, St. Louis, MO).

\section{Development of SPCL tubular scaffolds}

The SPCL tubular scaffolds were processed in a two-step methodological approach:

(1) Porous sheets featuring different pore geometries were produced by 3D plotting rapid prototyping (Bioplotter $^{\circledR}$; Envisiontec GmbH, Marl, Germany $)^{45,46}$ using two different interfilament orientations $\left(90^{\circ}\right.$ and $\left.45^{\circ}\right)$.
(2) Tubular scaffolds were obtained by rolling up porous sheets around a cylinder with subsequent heat treatment at $65^{\circ} \mathrm{C}$ for $30 \mathrm{~min}$ to induce the adhesion between filaments.

Up to six different porous tubular scaffold designs were obtained featuring a single or double layer combined with three different filaments' oriented relative to the tube main axis: filaments oriented at $0^{\circ}$ and $90^{\circ}$ relative to the tube main axis (90/1-2, further referred as $90 / 1$ and 90/2 for structures with one or two layers), filaments oriented at $0^{\circ}$ and $45^{\circ}$ relative to the tube main axis (45/1-2, further referred as $45 / 1$ and $45 / 2$ ), and filaments oriented at $45^{\circ}$ relative to the tube main axis $(X / 1-2$, further referred as $X / 1$ and $X / 2)$. As an example of the adopted scaffold references, processing combination $90 / 2$ refers to a double-layer scaffold featuring filaments orientated at $0^{\circ}$ and $90^{\circ}$ relative to the tube main axis, while $45 / 1$ refers to a single-layer scaffold featuring filaments orientated at $45^{\circ}$ relative to the tube main axis.

\section{Scanning electron microscopy}

Scanning electron microscopy was used for analysis of morphology/porosity of the different SPCL tubular scaffolds. For this purpose, all samples were coated with $\mathrm{Au} / \mathrm{Pd}$ via ion-sputtering before observation in a Stereoscan 360 scanning electron microscope (Leica Cambridge Co., Cambridge, United Kingdom).

\section{Micro-computed tomography}

The architecture and porosity of the tubular scaffolds were analyzed by micro-computed tomography ( $\mu-\mathrm{CT})$ using a desktop $\mu$-CT scanner (1072; SkyScan, Kontich, Belgium) at a voltage of $40 \mathrm{kV}$ and a current of $248 \mu \mathrm{A}$. Isotropic slice data were obtained by the system and reconstructed into $2 \mathrm{D} X Y$ slice images. Around 600 slice images per sample were compiled and subsequently employed in the rendering of 3D $\mathrm{XYZ}$ images to obtain quantitative architectural parameters. A $\mu$-CT analyzer and a $\mu$-CT volume realistic 3D Visualization software (SkyScan) was used as an image processing tool for reconstruction and creation/observation of 3D scaffold representations.

\section{Dynamic mechanical analysis}

Dynamic mechanical analysis was carried out using a uniaxial compression-loading scheme at $37^{\circ} \mathrm{C}$, under both dry and wet (phosphate buffered saline $0.1 \mathrm{M}, \mathrm{pH} 7.2$ ) conditions. Dynamic mechanical analysis was performed in a Tritec 2000B equipment (Triton Technology, Nottinghamshire, UK). The frequency ranged between 1 and $70 \mathrm{~Hz}$. Three replicates were conducted for each type of scaffold morphology.

\section{Development of the 3D hybrid systems}

The integration of gellan gum hydrogel into the central canal of the SPCL tubular scaffolds was performed by placing the tubular scaffolds into a specially designed mold, and by filling the empty volume with a gellan gum hydrogel as previously described by Oliveira et al. ${ }^{44}$ 


\section{In vitro cytotoxicity assessment}

To assess the short-term cytotoxicity of the developed scaffolds, the following guidelines described by Salgado et al. were used ${ }^{47}$ : minimum essential medium (MEM) extraction and MTS tests, both with 7, 14, 21, and 28 days of extraction period. In all tests material weight-to-extract fluid ratio was constant and equal to $0.2 \mathrm{~g} / \mathrm{mL}$ for porous samples, whereas for latex (negative control for cell viability) the ratio of material outer surface to extraction fluid was $2.5 \mathrm{~cm}^{2} / \mathrm{mL}$. After each period the extracts were filtered through a $0.45-\mu \mathrm{m}-$ pore-size filter. These assays are particularly suitable for assessing the possible toxic effect of leachables extracted from biomedical polymers. The objectives of the MEM extraction test are to evaluate changes in cell morphology and growth inhibition, whereas the MTS test determines whether cells are metabolically viable. In both cases, latex extracts (same extraction periods) were used as positive controls for cell death, while standard culture medium (formulation described bellow) was used as negative control for cell death.

Cell culture. The present experiment used a cell line of rat lung fibroblasts-L929, obtained from the European Collection of Cell Cultures. Cells were grown as monolayers in Dulbecco's modified Eagle's medium (DMEM; Sigma), supplemented with $10 \%$ fetal bovine serum(FBS; Gibco, Barcelona, Spain) and 1\% of an antibiotic-antimycotic mixture (Sigma). For the MEM extraction tests, cells were seeded in 12-well plates $\left(n=3,1 \times 10^{5}\right.$ cells/well), whereas for the MTS test, cells were seeded in 96-well plates $\left(n=3,1.8 \times 10^{4}\right.$ cells/well). In both cases cells were incubated for $24 \mathrm{~h}$ at $37^{\circ} \mathrm{C}$ in a $5 \%$ $(\mathrm{v} / \mathrm{v}) \mathrm{CO}_{2}$ cell culture incubator.

MEM extraction test. Twenty-four hours after cell seeding, the culture medium was removed from the wells and an identical volume, $1 \mathrm{~mL}$, of extraction fluid was added. Cultures were then incubated with the extracts for $72 \mathrm{~h}$, after which a live/dead assay was conducted by staining live cells with calcein-AM ( $1 \mathrm{mg} / \mathrm{mL}$; Molecular Probes, Eugene, OR) and nonviable cells with propidium iodide $(0.1 \mathrm{mg} / \mathrm{mL}$; Molecular Probes). Upon staining, cultures were observed under a fluorescence microscope (BX-61; Olympus, Hamburg, Germany).

MTS test. The metabolic viability of the L929 cells after exposure to extracts was determined by the bioreduction of [3-(4,5-dimethylthiazol-2-yl)-5-(3carboxymethoxyphenyl)2-(4-sulfophenyl)-2H-tetrazolium] (MTS; Promega, Madison, WI) into a brown formazan product by dehydrogenase enzymes. The extraction procedure was performed as previously described, using, in this turn, $200 \mu \mathrm{L}$ of extraction fluid per well. ${ }^{47}$ After $72 \mathrm{~h}$ of incubation, extracts were removed and a mixture of DMEM with MTS (5:1 ratio) was added to each well. Cells were then incubated for $3 \mathrm{~h}$ at $37^{\circ} \mathrm{C}$ in a $5 \%$ $\mathrm{CO}_{2}$ cell culture incubator after which optic density (OD) was determined at $450 \mathrm{~nm}$ using a 96-well plate reader (Tecan Sunrise, Männedorf, Switzerland).

\section{Histocompatibility evaluation}

Subcutaneous implantation. Young male Wistar rats (8 weeks old) were purchased from Charles River, housed in light- and temperature-controlled rooms, and fed a standard diet. The maintenance and manipulation of animals were in accordance with standardized Animal Care Guidelines. ${ }^{48}$ Hybrid 3D tubular scaffolds with one and two layers were implanted subcutaneously in the back of the animals for 7 , 14, 21, and 28 days. At the end of the implantation period, rats were sacrificed. The scaffolds were removed from the animals, fixed in 3.7\% formalin, embedded in Technovit 7100 (Kuzere, Wehrheim, Germany), and processed for hematoxylin-eosin staining.

\section{Hemisection SCI model}

Young male Wistar rats (8 weeks old) were purchased from Charles River, housed in light- and temperaturecontrolled rooms, and fed a standard diet. The maintenance and manipulation of animals were in accordance with standardized Animal Care Guidelines. ${ }^{48}$ To expose the spinal cord a laminectomy was performed at T8-T9 level. Once exposed, an unilateral defect was performed on the spinal cord, by removing approximately $2-3 \mathrm{~mm}$ of the latter. After this the 3D semi-tubular scaffolds were implanted in the defect site, with the SPCL phase aligned with the vertebral while the gellan gum was facing, and filling, the injured spinal cord. The scaffolds were removed from the animals, fixed in $3.7 \%$ formalin, decalcified, paraffin embedded, and finally processed for hematoxylin-eosin staining.

\section{Encapsulation assays}

For the cell encapsulation experiments within the gellan gum hydrogel, the human oligodendrocyte cell line MO3-13 was used. For this purpose, cells were grown as monolayer cultures in DMEM supplemented with 10\% FBS and 1\% antibiotic-antimycotic mixture until confluence. At this time cells were encapsulated in two different cellular densities (8 and $20 \times 10^{4}$ cells $/ \mathrm{mL}$ ) and injected into central canal of SPCL tubular scaffolds. Cell/scaffold constructs were then incubated in culture medium (DMEM, Gibco; 10\% FBS, Gibco; $1 \%$ antibiotic/antimycotic, Sigma) for 7 days at $37^{\circ} \mathrm{C}$ in a $5 \%$ $(\mathrm{v} / \mathrm{v}) \mathrm{CO}_{2}$ cell culture incubator. For this period, the distribution of viable cells was evaluated by confocal microscopy in combination with calcein-AM staining (Fluoview FV 1000; Olympus).

\section{Statistical analysis}

Statistical evaluation was performed using the one-way analysis of variance test followed by the Tukey posttest, to assess the statistical differences between groups in the porosity and cytotoxicity analyses. Evaluation of statistical differences on mechanical tests was performed using a twoway analysis of variance test followed by a Bonferroni posttest. Statistical significance was defined for $p<0.05$.

\section{Results and Discussion}

The objective of this work was to develop a novel biphasic 3D tubular structure composed of a biodegradable semirigid polymer and a hydrogel-like material. Comparing with other currently used strategies for scaffold-based SCI repair, our scaffold design has the advantages of combining the guidance stabilization from the 3D tubes, including the 
regeneration of vertebral bone, with the amenable conditions of a hydrogel-based material for cell and tissue migration. In this sense, our novel scaffold combines the beneficial effects of both strategies, overcoming the limitations of each strategy by itself. For instance, guidance tubes are able to stabilize the injured area from the mechanical point of view, while they act simultaneously as a physical guidance for SCI regeneration and protect the regenerating area from the invasion of adjacent connective tissue. ${ }^{2}$ However, often this guidance tubes lack the signaling cues, which, as described by Misgeld and coworkers, ${ }^{49}$ allow a more efficient cell and tissue migration along the injured area. Regarding hydrogels, it is known that they are quite versatile for SCI applications due to their cell encapsulation properties, peptide grafting, and growth factor loading. ${ }^{28}$ Nevertheless, in contrary to guidance tubes, hydrogels do not stabilize the injured area and have a limited capacity to avoid infiltration by the surrounding tissue. ${ }^{28}$ The chosen biomaterials for the development of the 3D bi-phasic scaffolds have also several others advantages. Both of them are polysaccharides prone to normal degradation in vivo, and both SPCL and gellan gum are easily integrated in different tissues. For instance, SPCL has been previously shown to allow the formation of bone extracellular matrix in vitro and bone in vivo, ${ }^{39,41}$ which is one of the main objectives of the SPCL phase under the concept herein presented. Further, this thermoplastic biomaterial is quite versatile from the processing point of view, allowing the use of rapid prototyping technologies for the development of the tubular structure. This is advantageous, as rapid prototyping (RP) allows the processing of highly reproducible scaffolds. On the other hand, gellan gum rapidly polymerize either at room temperature or at $37^{\circ} \mathrm{C}$, which allows the encapsulation of relevant cell populations ${ }^{50,51}$ without compromising their viability, as shown in the present article (see below). Moreover, gellan gum is quite amenable for chemical modification, and thus is makes possible to graft and align relevant peptides that can promote axonal migration, such as RGD and YISGS. For this purpose standard aqueous-phase peptide synthesis methodologies can be used on the polysaccharide backbone to promote adequate
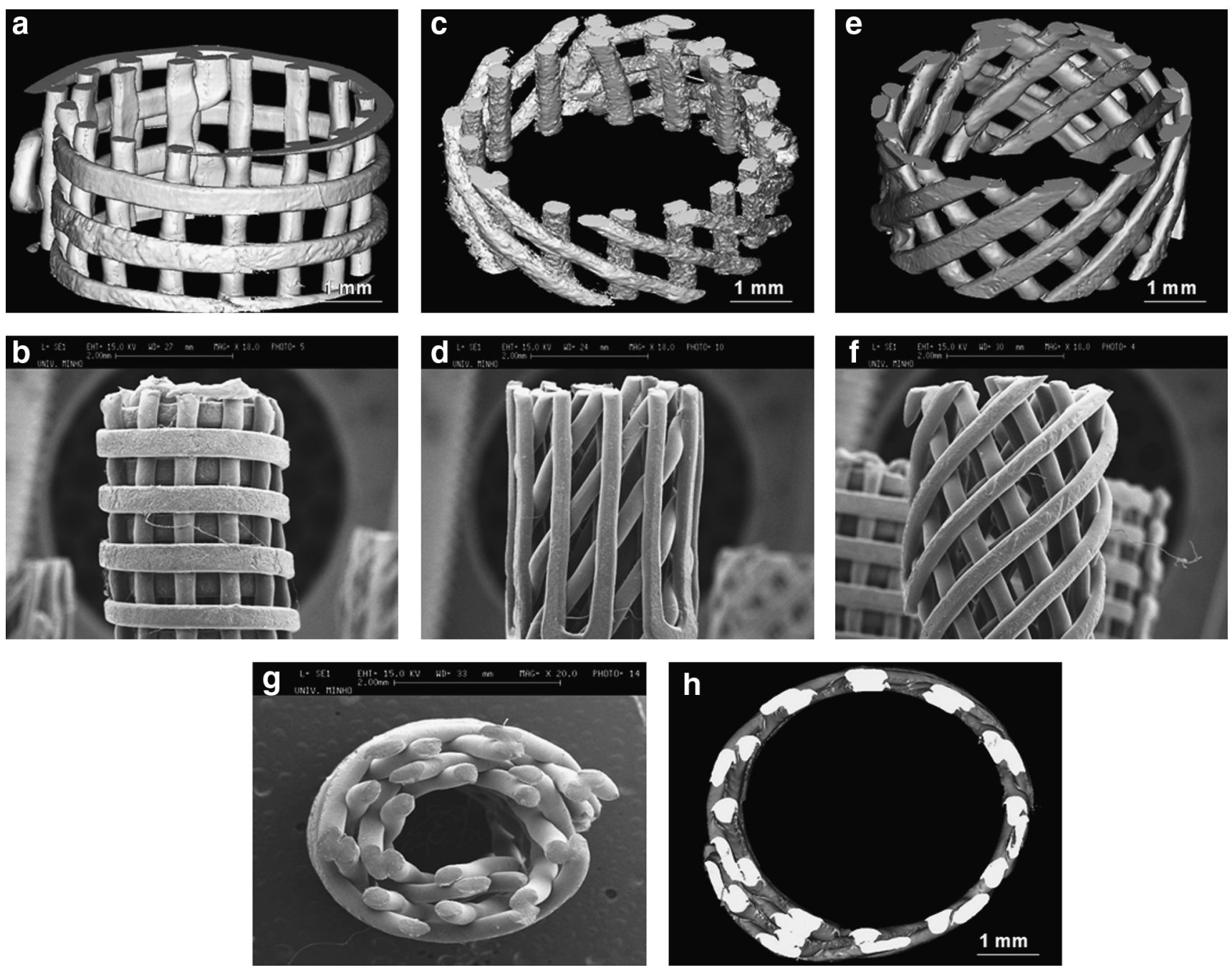

FIG. 1. Starch/poly- $\varepsilon$-caprolactone (SPCL) tubular scaffolds. (a) Micro-computed tomography ( $\mu$-CT) image of $90 / 1$ type structure. (b) Scanning electron microscopy (SEM) of 90/2 type structure. (c) $\mu$-CT image of 45/1 type structure. (d) SEM of $45 / 2$ type structure. (e) $\mu$-CT image of X/1 type structure. (f) SEM of X/2 type structure. (g) $\mu$-CT image of the top view of two-layer-type scaffolds. (h) SEM of the top view of one-layer-type scaffolds. 


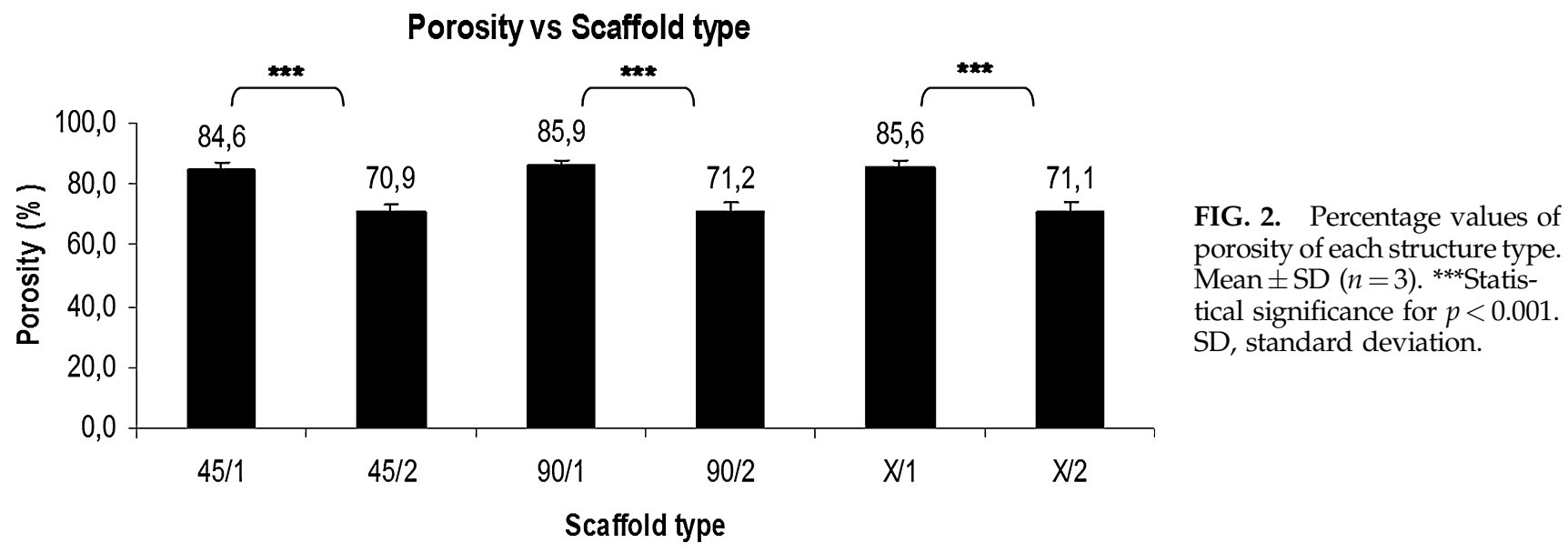

binding of the sequences of interest to the gellan gum. This can be achieved by activating the carboxylic groups of the sugar molecules with carbodiimide and then conjugating it with amino groups of methylester protected amino acids to which the presynthesized sequence of interest will be bound.
To develop the 3D guidance structure, a tubular scaffold was developed by combining rapid prototyping with a postprocessing thermal treatment. As it can be observed in Figure 1, the proposed processing methodology was quite versatile, allowing for the development of 3D tubular scaffolds a

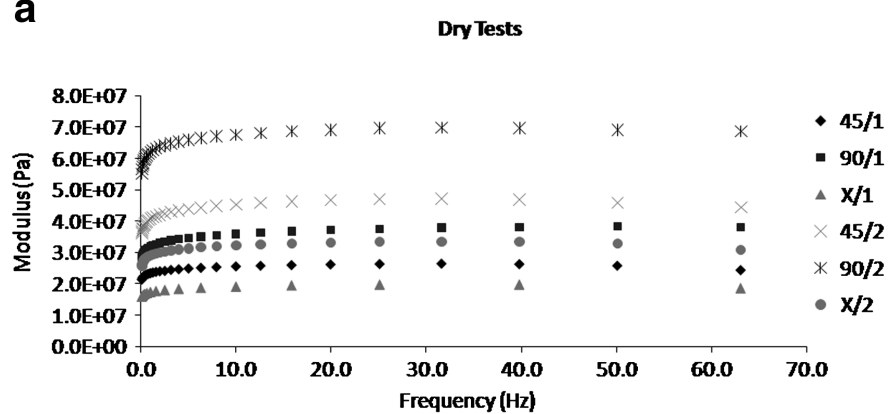

b

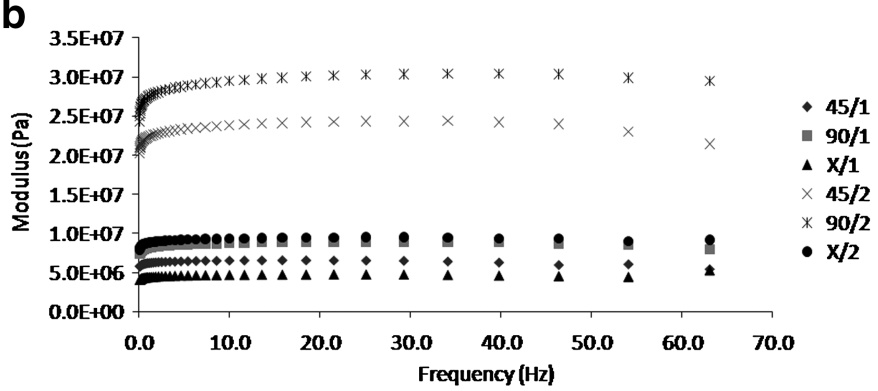

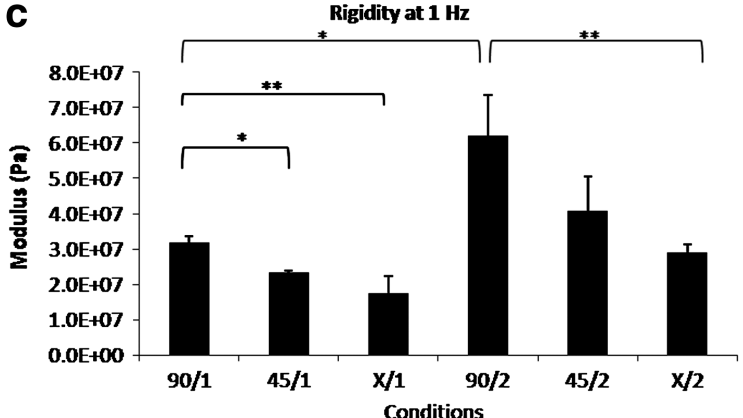

d

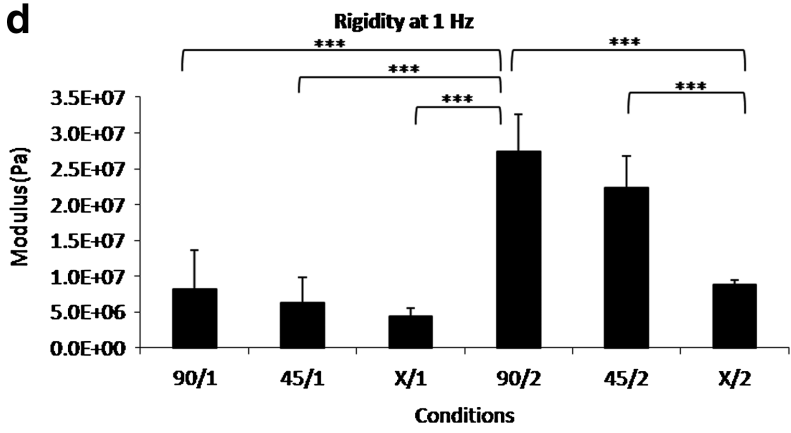

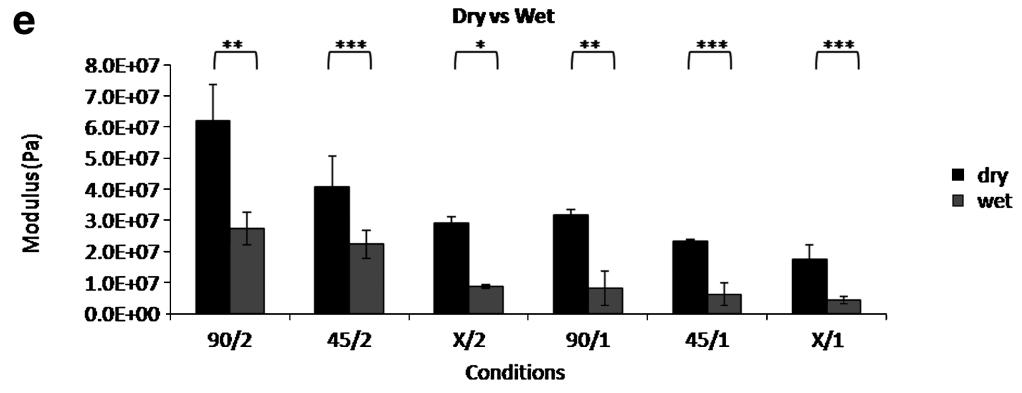

FIG. 3. Dynamic mechanical analysis of each scaffold type developed. (a) Dry compressive modulus evaluation in function of frequency. (b) Wet compressive modulus evaluation in function of frequency. (c) Rigidity comparison at $1 \mathrm{~Hz}$ in dry conditions. (d) Rigidity comparison at $1 \mathrm{~Hz}$ in wet conditions. (e) Comparison at $1 \mathrm{~Hz}$ between the dry and wet tests. Mean \pm SD $(n=3)$. Statistical significance for ${ }^{* *} p<0.001,{ }^{* *} p<0.01$, and ${ }^{*} p>0.05$. 

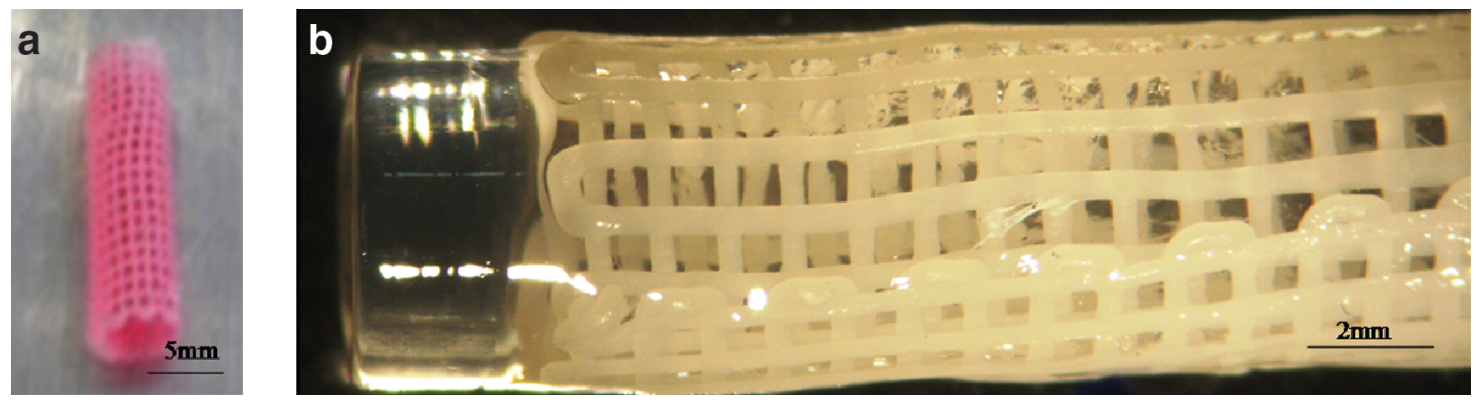

FIG. 4. First generation of functionalized multi supportive structure (FMSS). (a) After maintaining in culture with Dulbecco's modified Eagle's medium. (b) FMSS loupe view $(10 \times)$. Color images available online at www.liebertonline.com/ten.

with configurable outer layer thickness, fiber/filament orientation, and pore geometry. Fiber orientation ranged from $0^{\circ}$ to $90^{\circ}$ and resulted from the design adopted during the rapid prototyping stage.

Morphological analysis conducted by scanning electron microscopy and $\mu-\mathrm{CT}$ demonstrated that the developed processing technique allowed the production of structures with a precise tubular geometry with fully interconnected pore networks. Further analysis conducted using $\mu-C T$ revealed that scaffolds had fully interconnected network of pores with approximately $85 \%$ porosity for structures featuring a single outer layer and around $71 \%$ porosity for structures featuring double outer layers (Fig. 2). The differences in porosity observed were related to the outer thickness of the SPCL tubular structures, as the different orientations of the fibers did not affect the overall porosity of the scaffolds. The porosity values are in the range of those found in the literature for similar purposes. ${ }^{52}$

To assess their mechanical properties, the different tubular scaffolds were subjected to dynamic compression testing. The objective of these experiments was to evaluate their mechanical integrity and simultaneously determine their respective stiffness. Tests were carried out, at $37^{\circ} \mathrm{C}$ in dry and wet conditions, to simulate and study the influence of physiologic conditions on the mechanical properties of the tubular scaffolds, namely the influence of water absorption.
Moreover, to detect eventual mechanical collapse of the structure or layer detachment, the scaffolds were submitted to an increasing frequency test. Nevertheless, no major variation of storage modulus was detected upon the increase of frequency (Fig. 3a, b), which testifies the resilience of the scaffolds developed. Dry and wet tests revealed significant differences in the stiffness of the developed scaffolds. In the dry tests the $90 / 2$ samples obtained the highest value, followed in decreasing order of magnitude by structures $45 / 2$, $90 / 1, X / 2,45 / 1$, and $X / 1$ (Fig. 3c, $p<0,05$ ). Under wet conditions, as it would be expected, there was a decrease in stiffness. Consistent with the dry test observations, conditions $90 / 2$ and 45/2 had the highest values. However, in this case, the third condition that presented higher stiffness was X/2 followed by $90 / 1,45 / 1$, and X/1 (Fig. 3d). The stiffness of the different tubular scaffolds at $1 \mathrm{~Hz}$ under dry and wet conditions is plotted in Figure 3e. The differences observed were related with different fiber orientations and scaffold thicknesses. Scaffolds with two layers had higher stiffness values than the equivalent single-layer structures. On what concerns to fiber orientation, scaffolds with aligned fibers along the tube axis had higher resistance to compression force. Our results revealed that, $90 / 1$ and $90 / 2$, as well as $45 / 1$ and $45 / 2$ conditions contained higher stiffness values than those of $X / 1$ and $X / 2$ structures. For $90 / 1-2$ and 45/1-2 scaffolds, half of the fibers were aligned in the same direction
FIG. 5. MEM extraction evaluation of cell line L929 for four times of incubation $(7,14$, 21 , and 28 days) with three different conditions (positive control, negative control, and FMSS extract). Viable cells were staining with calceinAM and unviable cells staining with propidium iodide. Color images available online at www.liebertonline.com/ten.

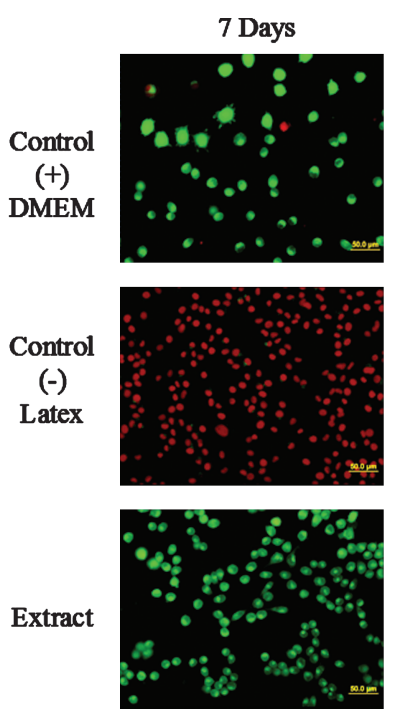

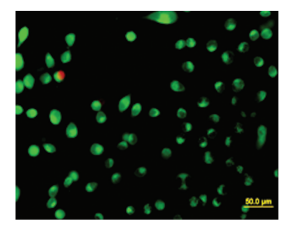
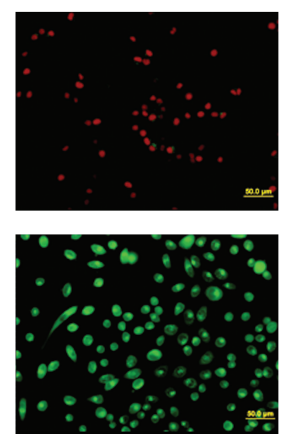

$21 \mathrm{D}$
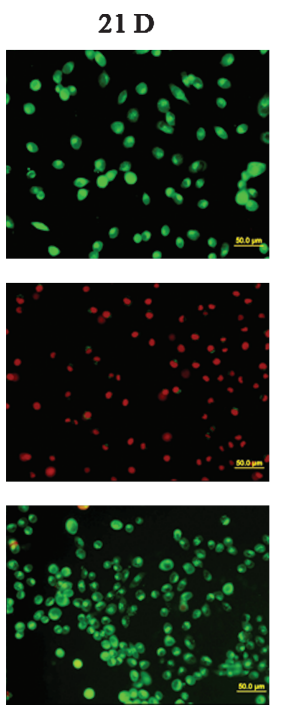

$28 \mathrm{D}$
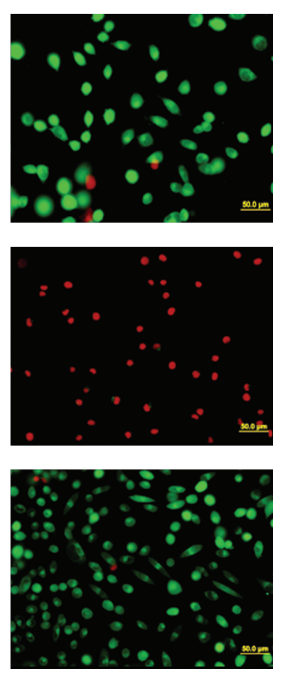


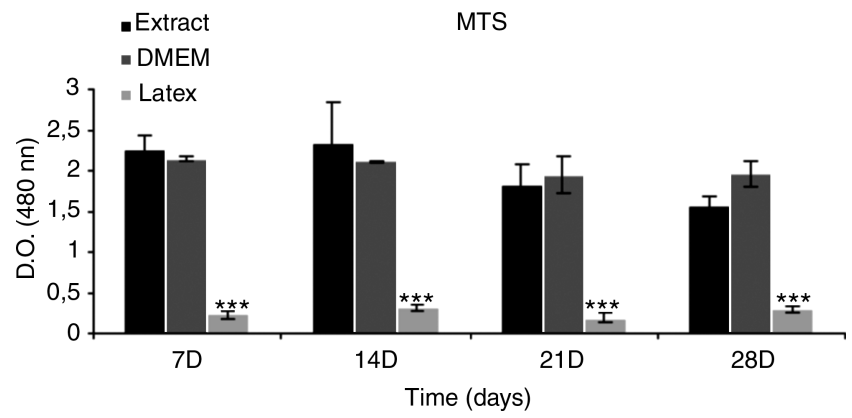

FIG. 6. MTS evaluation for cell line L929 for four times of incubation $(7,14,21$, and 28 days) with three different conditions (positive, Dulbecco's modified Eagle's medium [DMEM]; negative, Latex [controls and FMSS extract]).

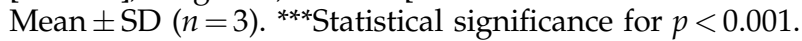

of the applied compression force. However, while for 90/1-2 scaffolds, approximately half of the fibers were aligned orthogonally to the compression force; for 45/1-2 conditions, the remaining half were diagonally aligned $\left(45^{\circ}\right.$ relative to the compression force). In theory, 45/1-2 and X/1-2 conditions should present higher stiffness than those experimentally observed, due to the stiffness contribution of diagonally orientated fibers-cosine $\left(45^{\circ}\right)$. The lower values of stiffness observed for scaffolds featuring diagonally orientated structures are probably due to buckling of the structure, which occurs upon compression due to geometrical instability of the structure. As previously shown (Fig. 3e), under wet conditions, all scaffolds exhibited a decrease in stiffness, which can be explained by the water absorption. Water acts as plasticicer of the polymer, reducing its resistance to plastic deformation. ${ }^{53}$ In SPCL, starch is the main water absorbent since PCL has low water uptake ability. ${ }^{53}$
Further, it could also be observed that the relative decrease in stiffness was larger in single-layer structures. This can be explained by the larger surface area/total volume ratio of single-layered structures as compared to double-layered structures. In double-layered scaffolds, the stacking of porous sheets reduces the relative surface area, which ultimately results in less water absorption. It is important to note that the mechanical tests with the SPCL structures were performed before gellan gum incorporation. Since the stiffness of gellan gum hydrogel is three orders of magnitude smaller than SPCL, no relevant mechanical effect would be expected for structures containing the hydrogel. Under dry conditions, the elastic modulus for gellan gum was determined to be $79.7 \pm 7.56 \mathrm{kPa}$, as characterized by Oliveira et al. ${ }^{44}$

Following the physical characterization of tubular scaffolds, the hydrogel phase was injected into the central canal of the tubes. This process allowed the formation of the biphasic structure presented in Figure 4. As it can be observed, the two phases were fully integrated in the system, being the central canal of the 3D SPCL tubular structure entirely filled with the gellan gum hydrogel. The main benefit of the proposed hybrid system arises from the individual potential advantages of each material. The SPCL frame is aimed at assuring the structural stiffness of the construct, while the gellan gum hydrogel is expected to create an amenable nerve regeneration environment.

Biological assays were performed upon the development of the hybrid SPCL/gellan gum structures. Their cytotoxicity was determined by MTS and MEM extraction tests on a L929 cell line, while cell encapsulation assays were carried out with an oligodendrocyte-derived cell line. Since the SCPL layer will be used to connect to adjacent bone, the stiffness of the SPCL tubular structures is very important, as they will
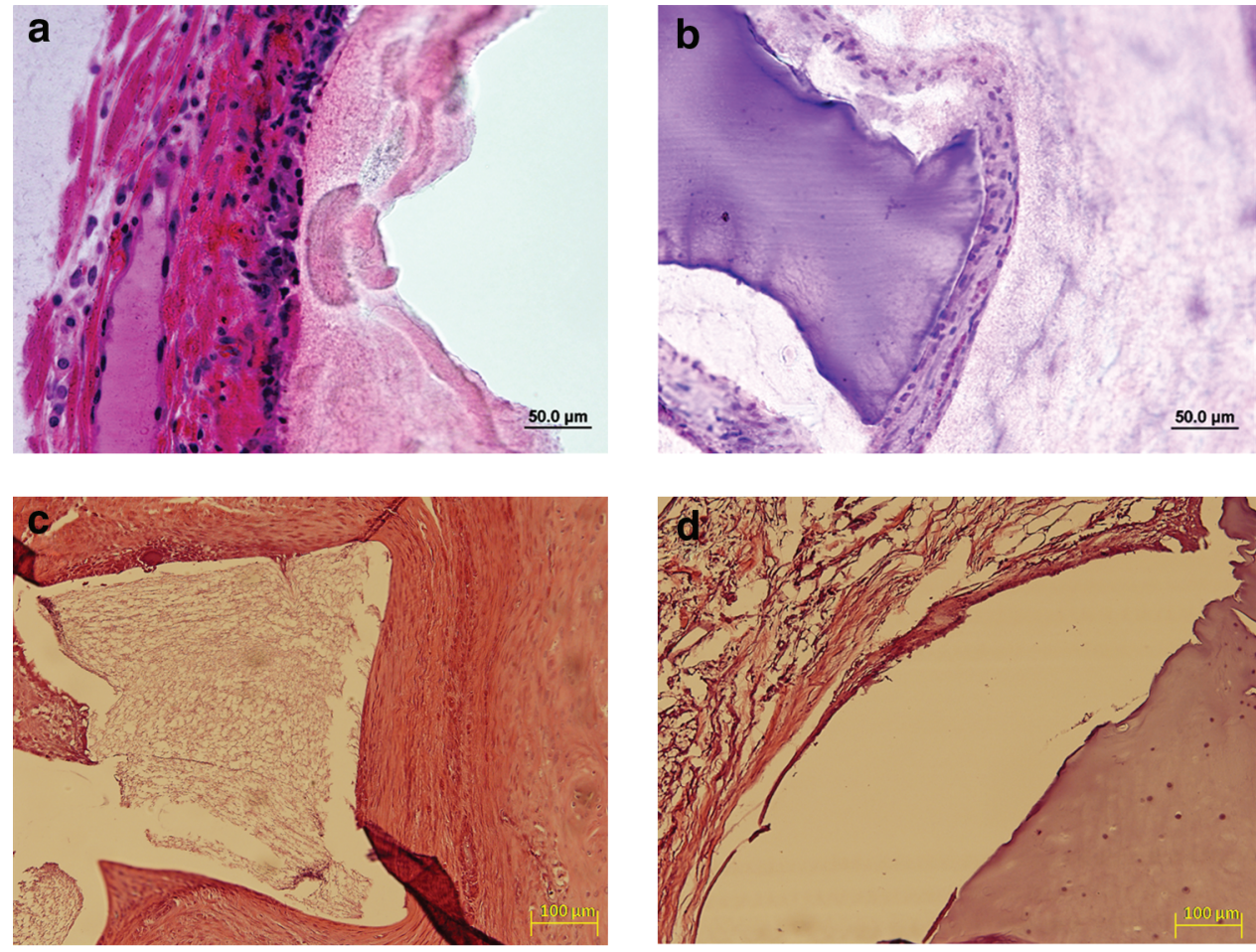

FIG. 7. Scaffolds were implanted in a subcutaneous $(\mathbf{a}, \mathbf{b})$ and hemisection $(\mathbf{c}, \mathbf{d})$ spinal cord injury model. In both cases histological sections were stained with hematoxylin-eosin. After 1 (a) and 4 (b) weeks of subcutaneous implantation, the tested hybrid scaffolds were well integrated within the surrounding tissue with minimal inflammatory response. Similar results were also obtained for the hemisection model (c, d), where it could be observed the formation of bone near SPCL struts. Color images available online at www .liebertonline.com/ten. 
FIG. 8. Direct contact evaluation of cell line M0III encapsulation on FMSS. The green points are oligodendrocytelike cells that appear dispersed on gellan gum matrix (a) and near the SPCL fibbers (b). Cells staining with calcein-AM. Color images available online at www .liebertonline.com/ten.
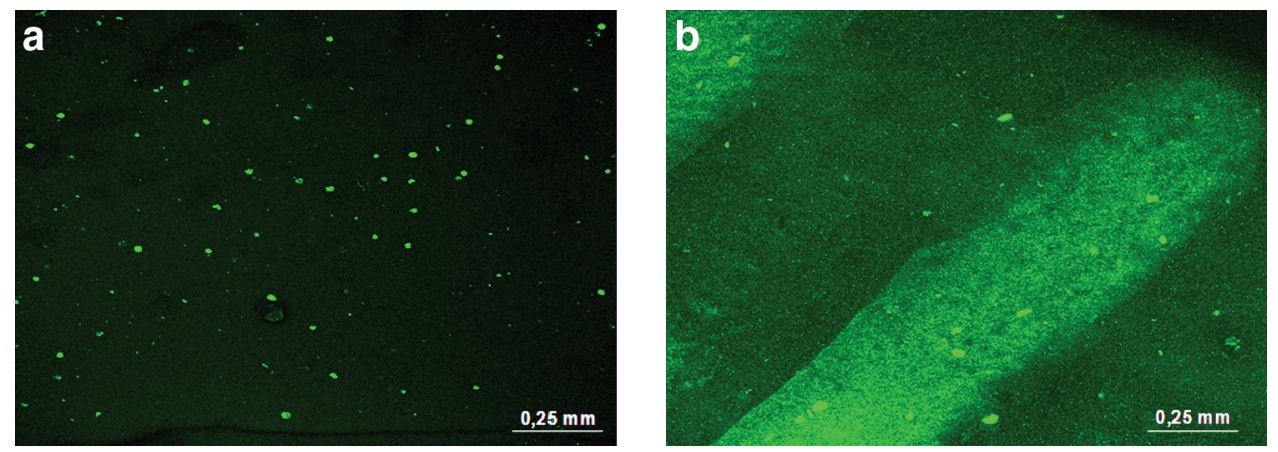

support directly the development of a mineralized tissue. In this sense, from all the developed structures, 90/2 samples were chosen for biological assays, as these present the best mechanical performance. Regarding cytotoxicity assays, MEM extraction tests associated with calcein/propidium iodide staining revealed that the extracts obtained from the hybrid 3D tubular structures did not cause cell death among L929 cells. The cell density, morphology, and viability were very similar between the extracted incubated cells and the cells grown in DMEM (positive control) (Fig. 5). These results were in contrast to cells grown in latex, and were then further confirmed by the MTS test (Fig. 6), under which L929 cells produced large amounts of a brown formazan product after incubation with the tested extracts. This is an indicator of normal metabolism as they were able to incorporate and metabolize MTS. MTS incorporation under these conditions was similar to the negative control and thus supportive of a lack of cytotoxic effect of these hybrid structures.

These favorable results were further confirmed by preliminary in vivo tests. In this case the 3D tubular hybrid structures were either subcutaneously implanted on Wistar rats (Fig. 7a, b) or in a hemisection model of SCI (Fig. 7c, d). In the subcutaneous model, rats developed an initial, but mild, inflammatory reaction detected after 1 week of implantation (Fig. 7a), which faded by the 4 th week (Fig. 7b), indicating a possible biocompatible character of the scaffolds. Moreover, we also observed no formation of fibrotic capsules, supportive of the lack of inflammatory response triggered by the implanted scaffolds. Finally, it was possible to confirm that pore size, geometry, and properties of the structure facilitated cells and connective tissue penetration within the scaffold. Similar results were also obtained for the SCI model, regarding the inflammatory response. As it can be seen in Figure 7c and d, no major inflammation processes were found. Moreover, it was possible to observe bone tissue (Fig. 7c) forming around SPCL struts. Nevertheless, the results of this preliminary experiment will be further confirmed in future experiments.

The interaction of central nervous system cell populations with the developed structures is extremely important since they will be one of the mediators of nerve regeneration induction within the hydrogel phase. Therefore, preliminary cell encapsulation assays were performed using the MO3-13 human oligodendrocyte cell line. The rationale for using these cells was based on future approaches that can be used, namely, the use of oligodendrocyte progenitors to foster remyelination repair in the affected SCI areas. As previously described, the cell population was first encapsulated within the gellan gum hydrogel before injection in the tubular structure. Confocal laser microscopy observation revealed that the hydrogel phase of the hybrid structure supported the in vitro culture of MO3-13 cells during the culturing period (up to 1 week). The cells were preferentially dispersed within the gellan gum matrix, being either at the center (Fig. $8 \mathrm{a}$ ) or at the vicinities (Fig. 8b) of the hydrogel.

\section{Conclusions}

With the present work it was possible to show that 3D plotting, a rapid prototyping technology, was successfully used in the development of starch-based scaffolds to be employed in future applications for SCI regeneration. We also showed that the developed SPCL tubular scaffolds possessed configurable mechanical performance, pore sizes, and interconnectivity values adequate for cell/tissue-based regenerative applications. We also demonstrated that the different orientations of the fibers and the number of layers in the developed structures influenced their mechanical properties. Moreover, we demonstrated that SPCL/gellan gum hybrid structures were noncytotoxic and disclosed an apparent biocompatible response in vivo. Finally, it was also possible to observe that these structures allowed the in vitro culture of oligodendrocyte-like cells for periods up to 1 week. Further work is ongoing using these hybrid systems in animal models, to determine their potential to therapeutic intervention of SCI, as well the functionality of improved of gellan gum hydrogels in terms of cell adhesion and guidance upon division.

\section{Acknowledgments}

Portuguese Foundation for Science and Technology through funds from Programa Operacional Ciência, Tecnologia, Inovação (POCTI) and/or Fundo Europeu de Desenvolvimento Regional (FEDER) programs (funding to ICVS, 3B's Research Group, predoctoral and postdoctoral fellowships to N.A. Silva, J.T. Oliveira, A.J. Salgado, and R.A. Sousa-SFRH/BD/40684/2007; SFRH/BD/17135/2004; SFRH/BPD/17595/2004; SFRH/BPD/17151/2004).

\section{Disclosure Statement}

No competing financial interests exist.

\section{References}

1. Sekhon, L.H.S., and Fehlings M.G. Epidemiology, demographics, and pathophysiology of acute spinal cord injury. Spine 15, S2, 2001. 
2. Samadikuchaksaraei, A. An overview of tissue engineering approaches for management of spinal cord injuries. J Neuroeng Rehabil 14, 15, 2007.

3. National Spinal Cord Injury Statistical Center. Spinal cord injury. Facts and figures at a glance. J Spinal Cord Med 28, 379, 2005.

4. DeVivo, M.J. Causes and costs of spinal cord injury in the United States. Spinal Cord 35, 809, 1997.

5. Ackery, A., Tator, C., and Krassioukov, A. A global perspective on spinal cord injury epidemiology. J Neurotrauma 21, 1355, 2004.

6. Winkler, T., Sharma, H.S., Gordh, T., Badgaiyan, R.D., Stalberg, E., and Westman, J. Topical application of dynorphin A (1-17) antiserum attenuates trauma induced alterations in spinal cord evoked potentials, microvascular permeability disturbances, edema formation and cell injury: an experimental study in the rat using electrophysiological and morphological approaches. Amino Acids 23, 273, 2002.

7. Bao, F., John, S.M., Chen, Y., Mathison, R.D., and Weaver, L.C. The tripeptide phenylalanine-(D) glutamate-(D) glycine modulates leukocyte infiltration and oxidative damage in rat injured spinal cord. Neuroscience 140, 1011, 2006.

8. Park, E., Velumian, A.A., and Fehlings, M.G. The role of excitotoxicity in secondary mechanisms of spinal cord injury: a review with an emphasis on the implications for white matter degeneration. J Neurotrauma 21, 754, 2004.

9. Conti, A., Cardali, S., Genovese, T., Di Paola, R., and La Rosa, G. Role of inflammation in the secondary injury following experimental spinal cord trauma. J Neurosurg Sci 47, 89, 2003.

10. Bracken, M.B., Shepard, M.J., Collins, W.F., Holford, T.R., Young, W., Baskin, D.S., Eisenberg, H.M., Flamm, E., LeoSummers, L., and Maroon, J. A randomized, controlled trial of methylprednisolone or naloxone in the treatment of acute spinal-cord injury. Results of the Second National Acute Spinal Cord Injury Study. N Engl J Med 322, 1405, 1990.

11. Bracken, M.B. Methylprednisolone and acute spinal cord injury: an update of the randomized evidence. Spine 26, S47, 2001.

12. Hurlbert, R.J. Methylprednisolone for acute spinal cord injury: an inappropriate standard of care. J Neurosurg 93, 1, 2000.

13. Bracken, M.B., and Holford, T.R. Neurological and functional status 1 year after acute spinal cord injury: estimates of functional recovery in National Acute Spinal Cord Injury Study II from results modeled in National Acute Spinal Cord Injury Study III. J Neurosurg 96, 259, 2002.

14. Houweling, D.A., Bar, P.R., Gispen, W.H., and Joosten, E.A. Spinal cord injury: bridging the lesion and the role of neurotrophic factors in repair. Prog Brain Res 117, 445, 1998.

15. Blesch, A., Lu, P., and Tuszynski, M.H. Neurotrophic factors, gene therapy, and neural stem cells for spinal cord repair. Brain Res Bull 57, 833, 2002.

16. Jones, L.L., Oudega, M., Bunge, M.B., and Tuszynski, M.H. Neurotrophic factors, cellular bridges and gene therapy for spinal cord injury. J Physiol 533, 83, 2001.

17. David, S., and Lacroix, S. Molecular approaches to spinal cord repair. Annu Rev Neurosci 26, 411, 2003.

18. Chau, C.H., Shum, D.K., Li, H., Pei, J., Lui, Y.Y., Wirthlin, L., Chan, Y.S., and Xu, X.M. Chondroitinase ABC enhances axonal regrowth through Schwann cell-seeded guidance channels after spinal cord injury. FASEB J 18, 194, 2004.

19. Wunderlich, G., Stichel, C.C., Schroeder, W.O., and Müller, H.W. Transplants of immature astrocytes promote axonal regeneration in the adult rat brain. Glia 10, 49, 1994.
20. Hikawa, N., Horie, H., and Takenaka, T. Macrophageenhanced neurite regeneration of adult dorsal root ganglia neurones in culture. Neuroreport 5, 41, 1993.

21. Ramón-Cueto, A., Cordero, M.I., Santos-Benito, F.F., and Avila, J. Functional recovery of paraplegic rats and motor axon regeneration in their spinal cords by olfactory ensheathing glia. Neuron 25, 425, 2000.

22. Myckatyn, T.M., Mackinnon, S.E., and McDonald, J.W. Stem cell transplantation and other novel techniques for promoting recovery from spinal cord injury. Transplant Immunol 12, 343, 2004.

23. Kakulas, B.A. Neuropathology: the foundation for new treatments in spinal cord injury. Spinal Cord 42, 549, 2004.

24. Syková, E., Jendelová, P., Urdzíková, L., Lesný, P., and Hejčl, A. Bone marrow stem cells and polymer hydrogelstwo strategies for spinal cord injury repair. Cell Mol Neurobiol 26, 1111, 2006.

25. Woerly, S., van Doana, D., Sosa, N., Vellis, J., and Espinosa, A. Reconstruction of the transected cat spinal cord following NeuroGel ${ }^{\mathrm{TM}}$ implantation: axonal tracing, immunohistochemical and ultrastructural studies. Int J Dev Neurosci 19, 63, 2001.

26. Hejčl, A., Lesný, P., Přádný, M., Michálek, J., Jendelová, P., Štulík, J., Syková, E. Biocompatible hydrogels in spinal cord injury repair. Physiol Res 57 Suppl 3, S121, 2008.

27. Tsai, E.C., Dalton, P.D., Shoichet, M.S., and Tator, C.H. Synthetic hydrogel guidance channels facilitate regeneration of adult rat brainstem motor axons after complete spinal cord transaction. J Neurotrauma 21, 789, 2004.

28. Salgado, A.J., Silva, N.A., Neves, N.M., Reis, R.L, and Sousa, N. Hydrogel based systems for spinal cord injury regeneration. In: Reis, R.L., ed. Natural-Based Polymers for Biomedical Applications. Cambridge, UK: Woodhead Publishing Limited, pp. 570-588, 2008.

29. Langer, R., and Vacanti, J.P. Tissue engineering. Science 260, 920, 1993.

30. Liu, S., Said, G., and Tadie, M. Regrowth of the rostral spinal axons into the caudal ventral roots through a collagen tube implanted into hemisected adult rat spinal cord. Neurosurgery 49, 143, 2001.

31. Yoshii, S., Ito, S., Shima, M., Taniguchi, A., and Akagi, M. Functional restoration of rabbit spinal cord using collagenfilament scaffold. J Tissue Eng Regen Med 3, 19, 2009.

32. Ying, L., and Shoichet, M. Light-activated immobilization of biomolecules to agarose hydrogels for controlled cellular response. Biomacromolecules 5, 2315, 2004.

33. King, V.R., Henseler, M., Brown, R.A., and Priestley, J.V. Mats made from fibronectin support oriented growth of axons in the damaged spinal cord of the adult rat. Exp Neurol 182, 383, 2003.

34. Taylor, S.J., McDonald, J.W., III, and Sakiyama-Elbert, S.E. Controlled release of neurotrophin-3 from fibrin gels for spinal cord injury. J Control Release 98, 281, 2004.

35. Reis, R.L., and Cunha, A.M. Characterization of two biodegradable polymers of potential application within the biomaterials field. J Mater Sci Mater Med 6, 786, 1995.

36. Cascone, M.G., Barbani, N., Cristallini Giusti, C., Ciardelli, G., and Lazzeri, L. Bioartificial polymeric materials based on polysaccharides. J Biomater Sci Polym Ed 12, 267, 2001.

37. Reis, R.L., Mendes, S.C., Cunha, A.M., and Bevis, M.J. Processing and in vitro degradation of starch/EVOH thermoplastic blends. Polym Intern 43, 347, 1997.

38. Malafaya, P.B., Elvira, C., Gallardo, A., San Román, J., and Reis, R.L. Porous starch-based drug delivery systems 
processed by a microwave route. J Biomed Sci Polym Ed 12, 1227, 2001.

39. Gomes, M.E., Sikavitsas, V.I., Behravesh, E., Reis, R.L., and Mikos, A.G. Effect of flow perfusion on the osteogenic differentiation of bone marrow stromal cells cultured on starchbased three-dimensional scaffolds. Tissue Eng 12, 801, 2006.

40. Reis, R.L., and Cunha, A.M. Starch Polymers. In: Buschow, K.H.J., Cahn, R.W., Flemings, M.C., Ilschner, B., Kramer, E.J., Mahajan, S., and Veyssière, P., Eds. Encyclopedia of Materials: Science and Technology, Maryland Heights, MO: Elsevier Science 2001, pp. 8810-8816.

41. Mendes, S.C., Bezemer, J., Claase, M.B., Grijpma, D.W., Bellia, G., Degli-Innocenti, F., Reis, R.L., de Groot, K., van Blitterswijk, C.A., and de Bruijn, J.D. Evaluation of two biodegradable polymeric systems as substrates for bone tissue engineering. Tissue Eng Suppl 1, S91, 2003.

42. Martins, A., Chung, S., Pedro, A.J., Sousa, R.A., Marques, A.P., Reis, R.L., and Neves, N.M. Hierarchical starch-based fibrous scaffold for bone tissue engineering applications. J Tissue Eng Regen Med 3, 37, 2009.

43. Salgado, A.J., Sousa, R.A., Fraga, J.S., Pego, J.M., Silva, B.A., Malva, J.O., Neves, N.M., Reis, R.L., and Sousa, N. Preliminary study on the effects of starch/polycaprolactone based blends aimed to be used for spinal cord injury regeneration in hippocampal neurons/glial cells viability and proliferation. J Bioact Compat Polym 24, 235, 2009.

44. Oliveira, J.T., Costa-Pinto, A.R., Martins, L., Malafaya, P.B., Sousa, R.A., Mano, J.F., Neves, N.M., and Reis, R.L. Gellan gum hydrogels as supports for human articular chondrocytes and human bone marrow cells for cartilage tissue engineering application. Abstract presented at ESF/EMBO Symposium Stem Cells in Tissue engineering isolation, culture, characterisation and applications, Sant Feliu de Guixols, Spain, S4SL09, 2006.

45. Yeong, W.Y., Chua, C.K., Leong, K.F., and Chandrasekaran, $M$. Rapid prototyping in tissue engineering: challenges and potential. Trends Biotechnol 22, 643, 2004.

46. Leong, K.F., Cheah, C.M., and Chua, C.K. Solid freeform fabrication of three-dimensional scaffolds for engineering replacement tissues and organs. Biomaterials 24, 2363, 2003.

47. Salgado, A.J., Coutinho, O.P., and Reis, R.L. Novel starch based scaffolds for bone tissue engineering: cytotoxicity, cell culture and protein expression. Tissue Eng 10, 665, 2004.
48. Van Zupten, L.F.M., Baumans, V., and Beynen, A.C. Principles of Laboratory Animal Science. Amsterdam: Elsevier, 2001.

49. Kerschensteiner, M., Schwab, M.E., Lichtman, J.W., and Misgeld, T. In vivo imaging of axonal degeneration and regeneration in the injured spinal cord. Nat Med 11, 572, 2005.

50. Oliveira, J.T., Gardel, L., Rada, T., Martins, L., Gomes, M.E., and Reis, R.L. Rabbit articular cartilage full-thickness size defects treated with novel gellan gum injectable hydrogels and autologous adipose stem cells. Tissue Eng A 2008. (Submitted).

51. Oliveira, J.T., Santos, T.C., Martins, L., Silva, M.A., Marques, A.P., Castro, A.G., Neves, N.M., and Reis, R.L. Performance of new gellan gum hydrogels combined with human articular chondrocytes for cartilage regeneration when subcutaneously implanted in nude mice. J Tissue Eng Regen Med 3, 493, 2009.

52. Moore, M.J., Friedman, J.A., Lewellyn, E.B., Mantila, S.M., Krych, A.J., Ameenuddin, S., Knight, A.M., Lu, L., Currier, B.L., Spinner, R.J., Marsh, R.W., Windebank, A.J., and Yaszemski, M.J. Multiple-channel scaffolds to promote spinal cord axon regeneration. Biomaterials 27, 419, 2006.

53. Alves, N.M., Saiz-Arroyo, C., Rodriguez-Perez, M.A., Reis, R.L., and Mano, J.F. Microhardness of starch based biomaterials in simulated physiological conditions. Acta Biomater 3, 69, 2007.

Address correspondence to: Nuno A. Silva, B.Sc.

3B's Research Group-Biomaterials, Biodegradables, and Biomimetics University of Minho

Headquarters of the European Institute of Excellence on Tissue Engineering and Regenerative Medicine AvePark, 4806-909 Taipas, Guimarães Portugal

Email: nsilva@dep.uminho.pt

Received: October 8, 2008 Accepted: July 16, 2009

Online Publication Date: September 1, 2009 